Analytica Chimica Acta, 72 (1974) 169-176

(1) Elsevier Scientific Publishing Company, Amsterdam - Printed in The Netherlands

\title{
THE ANODIC OXIDATION OF BASES IN THE SOLVENT m-CRESOL
}

M. BOS and E. A. M. F. DAHMEN

Department of Chemical Technology. Twente University of Technology. Enschede (The Netherlands)

(Received 11th February 1974)

Acid-base titrations can be carried out successfully in the solvent $m$ cresol $^{1}$. This is important for the detcrmination of the acid or base content of some polymers for which $m$-cresol is a good solvent. The study of acid-base coulometry in m-cresol was started because of the several advantages offered by coulometry: greater sensitivity, no problems with unstable titrants, and no need for standardization of titrants.

Preliminary experiments in which constant-current coulometric titrations were carried out in solutions of tetraethylammonium perchlorate in m-cresol showed that the electrochemical generation of base ( $m$-cresolate) at platinum electrodes does occur with $100 \%$ current efficiency. However, in these experiments, generation of acid did not occur with $100 \%$ current efficiency.

The present study of the anodic oxidation of bases in $m$-cresol was undertaken to establish conditions for $100 \%$ current efficiency in the generation of acid in $m$ cresol.

\section{EXPERIMENTAL}

\section{Chemicals}

$m$-Cresol (Merck "Zur Synthese") was purified by distilling (b.p. 201-202 ${ }^{\circ} \mathrm{C}$ ), drying for $24 \mathrm{~h}$ on molecular sieve ( $3 \AA$, Union Carbide) and distilling again (b.p. $201-202^{\circ} \mathrm{C}$ ). The second distillate was used within a day. Some batches purified by this method still had a detectable acid or base content. Only those batches were used that showed no detectable amount of acid or base in acid-base titration and had a range from -1.55 to $+0.7 \mathrm{~V}$ in polarography with tetraethylammonium perchlorate as supporting electrolyte.

Sodium cresolate solutions were prepared by dissolving the appropriate amount of sodium in $m$-cresol.

Tetraethylammonium perchlorate (TEAP; Eastman, reagent grade) was recrystallized from ethanol.

1,3-Diphenylguanidine (Eastman, reagent grade), tetramethylammonium chloride (Eastman, reagent grade), tetramethylguanidine (Eastman, pract. grade), morpholine (Merck, "Zur Synthese"), strychnine (Merck, purum), triethylamine (Koch-Light, puriss.), n-butylamine (Fluka, puriss.) and mercury (Drijhout, polarographic grade) were used as received. 


\section{Apporatus}

A Radiometer polarograph type PO4, equipped with a Sargent IR compensator model $A$, was used. The polarographic cell was thermostatted at $20 \pm 0.01^{\circ} \mathrm{C}$, and a three electrode system was used. The reference electrode $(\mathrm{Ag} / \mathrm{AgCl})$ in $0.1 \mathrm{M}$ tetramethylammonium chloride in $m$-cresol was connected via a salt bridge with $0.1 M$ tetraethylammonium perchlorate in $m$-cresol to the test solution. The auxiliary electrode was a mercury pool. The characteristics of the DME were: h. $56 \mathrm{~cm}: \mathrm{m}, 2.87 \mathrm{mg} \mathrm{s}^{-1}: 1,2.70 \mathrm{~s}$ (open circuit in $0.1 \mathrm{M}$ TEAP in m-cresol).

For the rotating disc electrode equipment, the indicator electrode was a Tacussel platinum ring/disc electrode with a driving mechanism (type Asservitex) of which the ring section was not used. The area of the disc was $8.04 \mathrm{~mm}^{2}$. The potentiostat was a Bipad type from Tacussel. The reference electrode, salt bridge

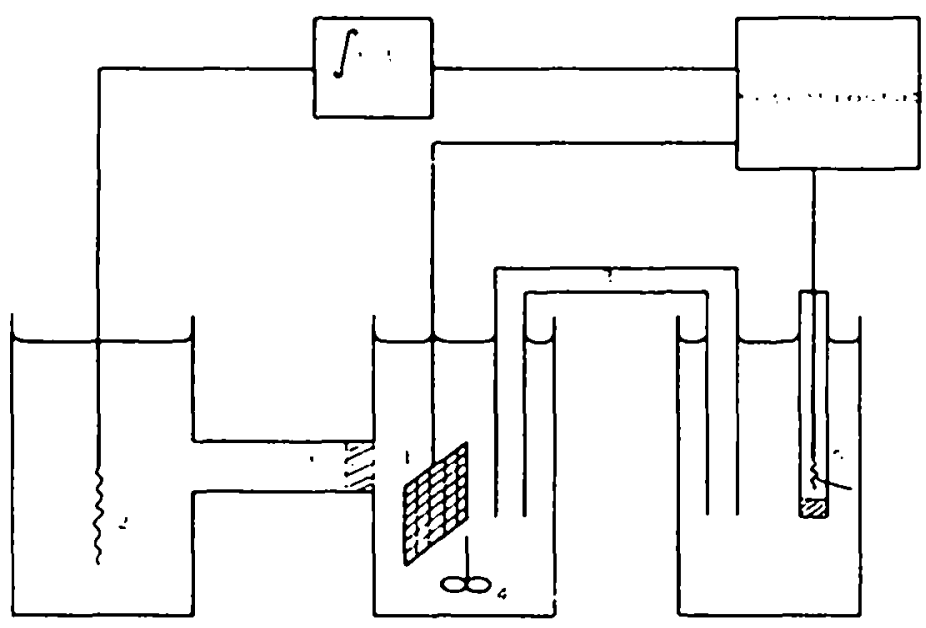

Fig. 1. Apparattus for constant-potential coulometry.

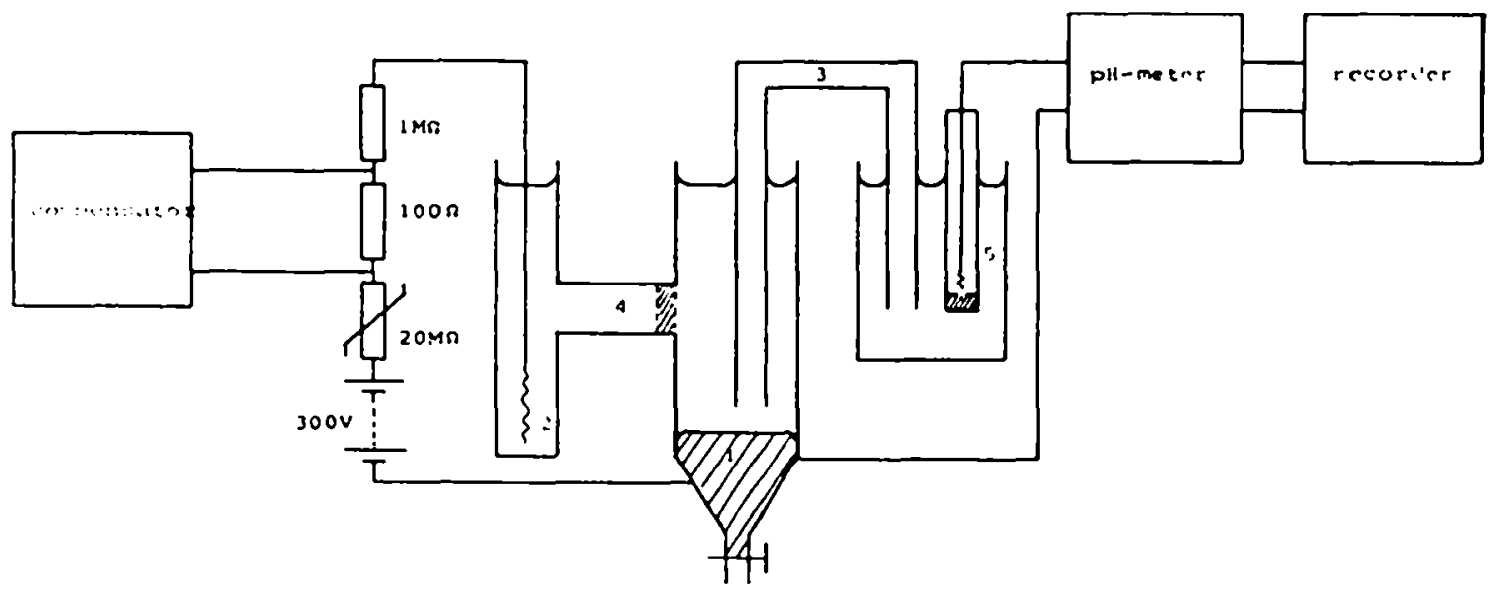

Fig. 2. Apparatus for chronopotentiometry. 
and auxiliary electrode were as described above. Measurements were carried out at room temperature.

For the constant-potential coulometric equipment, an H-type cell was operated at room temperature; the compartments of the cell were separated by a G-4 glass frit. A Tacussel Bipad potentiostat and an electronic integrator constructed from a Keithley operational amplifier, type K301, were used. The platinum gauze working electrode had an area of $4 \mathrm{~cm}^{2}$, and the auxiliary clectrode was a platinum wire. The reference electrode was $\mathrm{Ag} / \mathrm{AgCl}$ in $0.1 \mathrm{M}$ tetramethylammonium chloride in $m$-cresol, and was connected to the test solution via a $0.1 M$ TEAP in $m$-cresol salt bridge. The arrangement is shown in Fig. 1.

For the chronopotentiometry equipment, the H-type cell was again operated at room temperature, and a G-4 glass frit separated the compartments. The working electrode was a mercury pool (area, $3.87 \mathrm{~cm}^{2}$ ) at the bottom of one compartment. The auxiliary electrode was a platinum wire in the other compartment. The reference electrode was an $\mathrm{Ag} / \mathrm{AgCl}$ electrode in $0.1 \mathrm{M}$ tetramethylammonium chloride in m-cresol: this electrode was connected to the test solution via a salt bridge of $0.1 M$ TEAP in m-cresol. The outlet of this salt bridge was brought close to the surface of the mercury pool. The circuit consisted of a $300-\mathrm{V}$ stabilized power supply (v.d. Heem, type 8619), a large resistor, a Knick pH meter type PH23, and a Philips recorder type PM8100. The exact current was measured across a 100-ohm precision resistor in the circuit with a compensator galvanometer (Bleeker, type 2164). A schematic diagram is given in Fig. 2.

Procedures

Polarography. As supporting electrolyte $0.1 M$ TEAP was used. Stock $0.01 M$ solutions (accurately standardized by titration) of the bases were used to prepare dilution series in the concentration range $5 \cdot 10^{-4} M$ to $1.5 \cdot 10^{-3} M$ (in $0.1 M$ TEAP in $m$-cresol).

These solutions were placed in the polarographic cell and deoxygenated by bubbling nitrogen (purified over copper at $400^{\circ} \mathrm{C}$ ) through the solution for $20 \mathrm{~min}$. The polarographic curves were recorded without damping at a speed of $0.2 \mathrm{~V}$ $\min ^{-1}$, with the sensitivity at $5 \mu \mathrm{A}$ full scale.

Constant potential coulometry. Into the compartment of the working clectrode was placed $25 \mathrm{ml}$ of a solution of $0.1039 M \mathrm{n}$-butylamine in $1 M$ TEAP, and the other compartment was filled with $1 M$ TEAP. With the potentiostat the working electrode was maintained at $+0.650 \mathrm{~V}$. The solution in the working electrode compartment was stirred. After $10 \mathrm{~h}$, the electrolysis was stopped, the intcgrator was read and a sample was taken, which was titrated for n-butylamine content.

Voltammetry at the rotating platinum disc electrode. Voltammetric curves were recorded by measuring the disc current versus the disc voltage, for different rotating speeds of the electrode in a solution of $10^{-3} M$ n-butylamine in $0.1 M$ TEAP. Oxygen was removed by bubbling nitrogen through the solution for $20 \mathrm{~min}$.

Chronopotentiometry. As supporting electrolyte $0.1 M$ TEAP was used. From a stock solution of $0.01 M$-butylamine in $0.1 M$ TEAP in $m$-cresol, dilutions were made with $0.1 M$ TEAP to give concentrations in the range $5 \cdot 10^{-4} M$ to $2 \cdot 10^{-3} \mathrm{M}$. These solutions were placed in the cell. The chronopotentiogram was recorded after the solutions had been deoxygenated by bubbling nitrogen for $20 \mathrm{~min}$. 
When current reversal chronopotentiograms were recorded, the current was reversed before the occurrence of the transition.

\section{RESULTS}

\section{Polarography}

The polarographic oxidation waves of all the nitrogen bases investigated and of sodium cresolate all show the same characteristics. A typical example is the oxidation wave of sodium m-cresolate (Fig. 3). The slope of the graph of $E_{\mathrm{DME}}$ versus $\log \left(i /\left(i_{\mathrm{d}}-i\right)\right)$ is $82 \mathrm{mV}$ in this case, showing that the oxidation is irreversible. For morpholine, the influence of the height of the mercury column on the diffusion current was determined. The diffusion current versus the square root of the mercury height was linear (Fig. 4), indicating that the oxidation of morpholine is diffusioncontrolled.

The compounds tetramethylguanidinc,strychnine and n-butylamine gave a socalled "prewave" preceding the normal oxidation wave. The height of this prewave was independent of concentration. while the total height of the prewave and normal
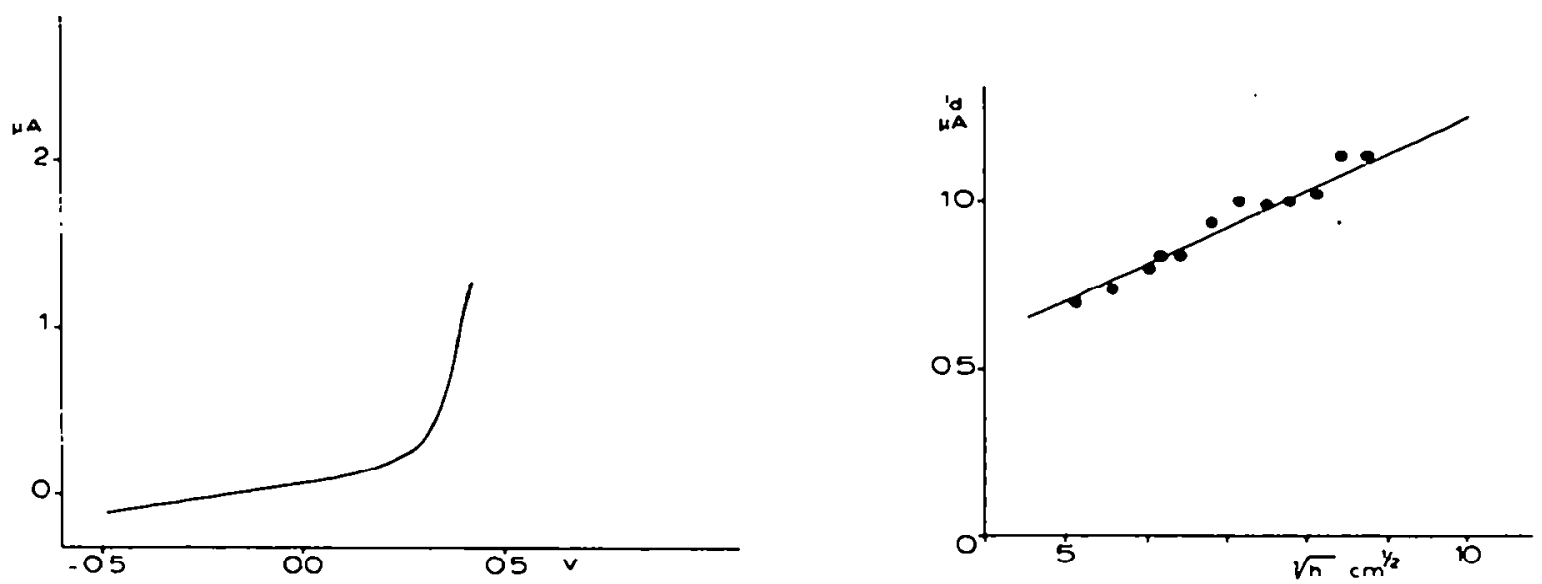

Fig. 3. Polarographic curve for $1.5 \cdot 10^{-9} \mathrm{M}$ sodium $m$-cresolate in $0.1 \mathrm{M}$ TEAP in $\mathrm{m}$-cresol.

Fig. 4. Diffusion current for the anodic oxidation wave of $10^{-3} M$ morpholine in $0.1 M$ TEAP in m-cresol versus (mercury height)t.

\section{TABLE I}

TOTAL WAVE HEIGHT AND HEIGHT OF PREWAVE FOR THE POLAROGRAPHIC OXIDATION OF STRYCHNINE

\begin{tabular}{cll}
\hline Concn. $(M)$ & is tolul wave $(\mu A)$ & is preware $(M A)$ \\
\hline $5 \cdot 10^{-4}$ & 0.48 & 0.19 \\
$7.5 \cdot 10^{-4}$ & 0.68 & 0.18 \\
$10^{-3}$. & 0.90 & 0.17 \\
$1.5 \cdot 10^{-3}$ & 1.40 & 0.17 \\
\hline
\end{tabular}


TABLE II

POLAROGRAPHY OF BASES IN $M$-CRESOL

(Supporting elcctrolytc 0.1 M TEAP)

\begin{tabular}{|c|c|c|c|}
\hline$C$ (m)mpenmel & $E_{1}\left(V^{\prime}\right.$ es. $\left.A g / A \eta C l\right)$ & $I_{\alpha}\left(\mu A M M^{-1} m g^{-1} s^{1}\right)$ & pK. \\
\hline Dimethyl:ıminouzobenzene & 0.52 & 0.398 & 11 \\
\hline Sirychninc & 0.43 & 0.388 & 13 \\
\hline n-13utylaminc & 0.41 & 0.419 & 13.9 \\
\hline Morpholine & 0.40 & 0.408 & 12.1 \\
\hline Tricthylaminc & 0.39 & 0.395 & 14.2 \\
\hline Sodium 11 -cresolitte & $0.38^{3}$ & 0.398 & - \\
\hline 1.3-Diphenylgua nidine & 0.38 & 0.376 & - \\
\hline Tetramethylguanidine & 0.38 & 0.389 & 14.5 \\
\hline
\end{tabular}

wave was related to the concentration (see Table I). Most likely this prewave is due to adsorption ${ }^{2}$.

The polarographic results are summarized in Table 11 . A comparison of the $E_{\ddagger}$ values for the oxidation of the bases with their $\mathrm{p} K_{\mathrm{a}}$ values in $m$-cresol (Table II) shows that a strong base is oxidized more easily than a weak base. This can be explained if it is assumed that in fact the m-cresolate ion is oxidized, because the concentration of free cresolate ions is determined by the equilibrium constants of the reaction

$$
\mathrm{B}+\mathrm{HCres}=\mathrm{BH}^{+} \mathrm{Cres}^{-} \leftrightharpoons \mathrm{BH}^{+}+\mathrm{Cres}^{-}
$$

From Table II it can be seen that the diffusion current constants $\left(I_{d}\right)$ are about the same for all bases. The mean value is $0.396 \mu \mathrm{A} \mathrm{mM}-1 \mathrm{mg}^{-1} \mathrm{~s}^{ \pm}$.

The diffusion constant can be calculated from the DME characteristics $m^{3} t^{\frac{1}{*}}=2.383 \mathrm{mg}^{+} \mathrm{s}^{-t}$, and the Ilkovic equation $i=607 \mathrm{nD}^{ \pm} \mathrm{cm}^{4} t_{\max }^{ \pm}$. The value was found to be $4.2 \cdot 10^{-7} \mathrm{~cm}^{2} \mathrm{~s}^{-1}$, if a one-electron transfer is assumed.

Oxilation of 1 -butylamine in $m$-cresol at the rotating platinum disc electrode

The current-voltage curves for the oxidation of a solution of $10^{-3} \mathrm{M}$

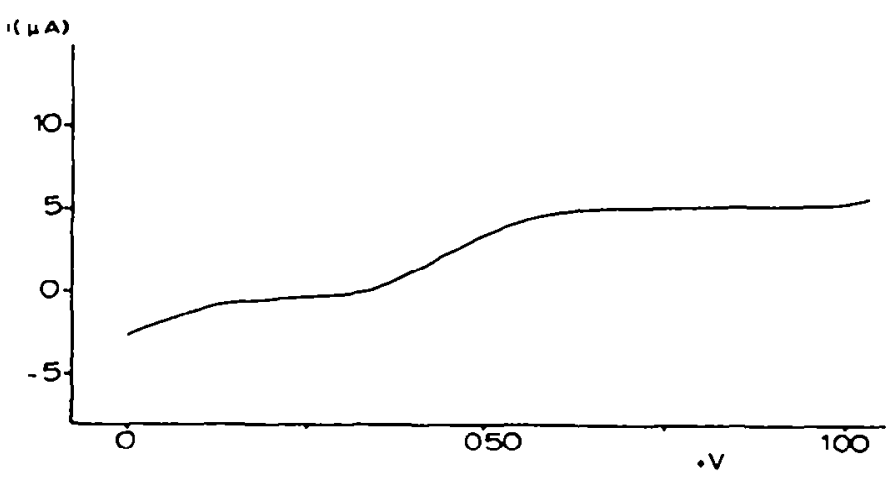

Fig. 5. Oxidation of $10^{-3} M$ n-butylamine in $0.1 M$ TEAP in m-cresol at the rotating platinum disc at 1800 r.p.m. 

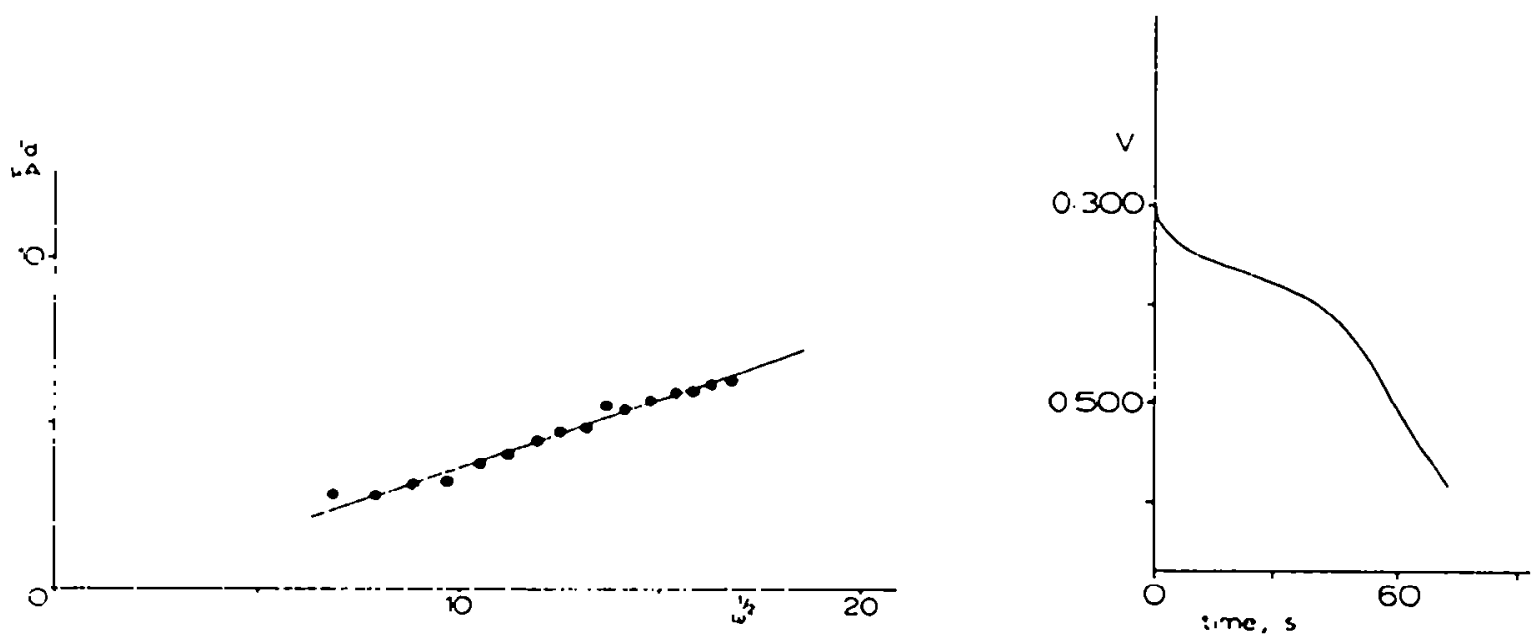

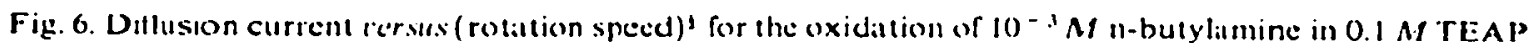
in m-cresol all the rotating plattinum disc.

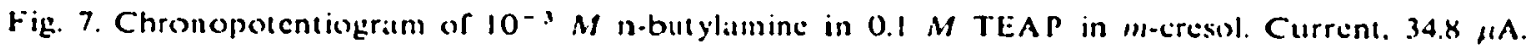

$\mathrm{n}$-butylamine in $0.1 M$ TEAP in $m$-cresol were recorded at different rotating speeds of the disc. A typical voltammetric curve is shown in Fig. 5 . It can be seen that oxidation of either the solvent or the supporting electrolyte starts at about $+0.95 \mathrm{~V}$. From the curves, the limiting current at different rotalting speeds was determined: Fig. 6 shows the graph of the limiting currents versiss the square root of the rotating speed. With the Levich equation, $i=0.62 \| F A D^{t} v^{-l}(1)^{t} c$, the diffusion coefficient can be calculated from the slope of this graph. With the kinematic viscosity $\mathrm{l}=0.2013$, the value $4.4 \cdot 10^{-7} \mathrm{~cm}^{2} \mathrm{~s}^{-1}$ was found for the diffusion coefricient. This is in good agreement with the value $4.2 \cdot 10^{-7}$ found from the Ilkovic cquation in the polarographic determination.

\section{Coulometry at constant potential}

After $10 \mathrm{~h}$ of electrolysis of $25 \mathrm{ml}$ of a solution containing $0.1039 \mathrm{M} \mathrm{n}$ butylamine in $1 \mathrm{M}$ TEAP. $212.9 \mu \mathrm{eq}$ of electrical charge hald passed through the cell. In this time, the titer of the n-butylamine solution decreased to $0.0950 \mathrm{M}$. corresponding to a conversion of $222.5 \mu$ mole of n-butylamine. This confirms the one-electron transfer already found in polarography and voltammetry at the rotating disc electrode.

\section{Chronopotentiometry}

Chronopotentiograms were recorded for solutions of n-butylamine in $0.1 M$ TEAP in $m$-cresol in the concentration range $5 \cdot 10^{-4} M$ to $2 \cdot 10^{-3} M$. The current was between 56.8 and $26.9 \mu \mathrm{A}$. A typical chronopotentiogram is given in Fig. 7 . The results of the experiments are given in Table III, which shows that the product $i t^{t}$ is constant. indicating that the oxidation of n-butylamine in $m$-cresol is diffusion-controlled. 
TABLE III

CHRONOPOTENTIOMETRY OF N-BUTYLAMINE IN 0.1 M TEAP IN M-CRESOL“

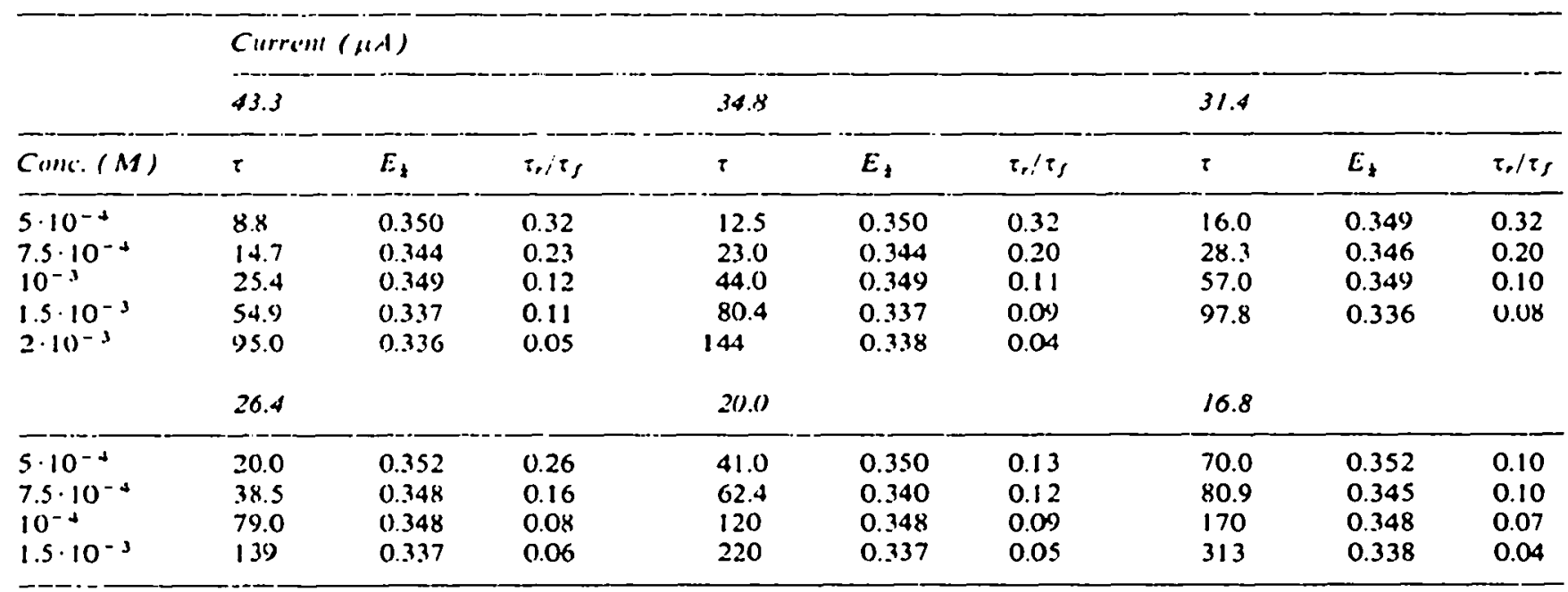

" $r$ in $s: E_{q}$ in $V$ versus $\mathrm{Ag} / \mathrm{AgCl} ; \tau_{, .}$transition time reduction: $\tau_{p}$ transition time oxidation.

The value $\partial E_{\ddagger} / \hat{r} \ln i_{0}$ at constant concentration is approximately zero, whereas $\delta E_{f} / \hat{i}$ In $c$ at constant current is negative. The ratio of the transition time for the reduction to the transition time for the oxidation when current reversal is applied, is dependent on bulk concentration and becomes less when the bulk concentration is increased.

Comparison of these facts with the diagnostic criteria for various kinetic schemes in chronopotentiometry given by Reinmuth ${ }^{3}$, indicates that the electron transfer is followed by a chemical reaction of higher than first order.

\section{DISCUSSION}

When a base $\mathrm{B}$ is dissolved in m-cresol the following equilibria are established:

$$
\mathrm{B}+\mathrm{HCres} \leftrightharpoons \mathrm{BH}^{+} \mathrm{Cres}^{-} \leftrightharpoons \mathrm{BH}^{+}+\mathrm{Cres}^{-}
$$

From the fact that the oxidation of bases in $m$-cresol is diffusion-controlled and that the $E_{\ddagger}$ values decrease with increasing $p K_{\mathrm{a}}$ values for different bases, it can be concluded that the m-cresolate ion is the electroactive species and that the equilibria between free base, ion pair and m-cresolate ion are rapid. As the experiments show that the oxidation is a one-electron process, its initial product must be the phenoxy radical.

Chronopotentiometry indicated that this phenoxy radical is subject to a further chemical reaction of higher than first order. For the phenoxy radical, different resonance structures are given ${ }^{4}$ :<smiles>CCOCCOc1cccc(C=Cc2ccc(C)cc2C)c1</smiles> 
I hese radicals call react to give dimers:

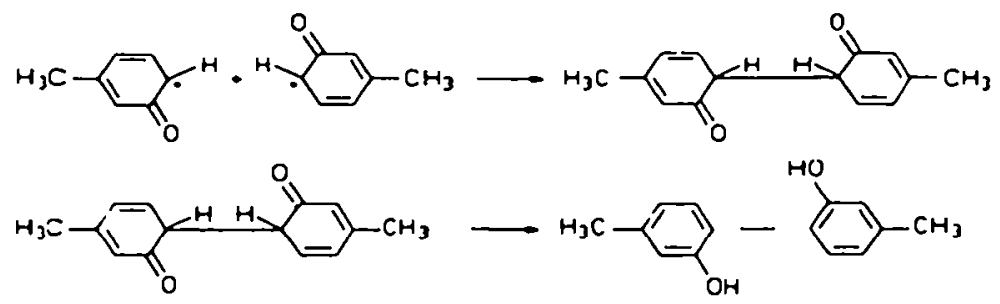

It is most likely that the electrochemical step is followed by a dimerization.

The authors thank Mrs. J. W. Th. Siemerink for her experimental help. Mrs. A. Nijkamp-Peterink for preparing the manuscript. and $\mathrm{Mr}$. R. H. Arends for making the drawings.

\section{SUMMARY}

The oxidation of bases in the solvent m-cresol was investigated by polarography, voltammetry at the rotating plat inum disc electrode and by chronopotentiometry. It was shown that the m-cresolate ion is the electroactive species in this reaction. The oxidation is a one-electron process giving a phenoxy radical. This phenoxy radical is converted in a chemical reaction of higher than first order, most likely to the dimer.

\section{REFERENCES}

1 M. Bos and E. A. M. F. Dahmen. Alul. Chim. Acta. 57 (1971) 361.

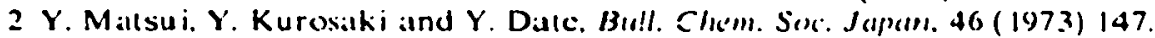

3 W. H. Reinmuth. Anal. Chem.. 32 (1960) 1514

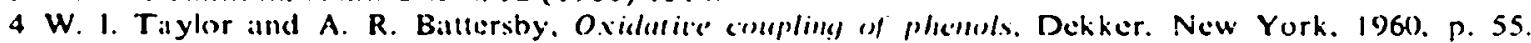

